\title{
Conversion of a murine monoclonal antibody A13 targeting epidermal growth factor receptor to a human monoclonal antibody by guided selection
}

\author{
Ki-Hwan Chang, Min-Soo Kim, Gwang-Won Hong, \\ Yong-Nam Shin and Se-Ho Kim ${ }^{1}$
}

\author{
Antibody Engineering Lab \\ Central Research Center, Green Cross Corp. \\ Yongin 446-770, Korea \\ ${ }^{1}$ Corresponding author: Tel, 82-31-260-9805; \\ Fax, 82-31-260-9020; E-mail, sehokim @greencross.com \\ http://dx.doi.org/10.3858/emm.2012.44.1.005
}

Accepted 8 November 2011

Available Online 8 November 2011

Abbreviations: HAMA, human anti-mouse antibody; scFv, single chain $\mathrm{Fv}$; $\mathrm{V}_{\mathrm{H}}$, variable region of immunoglobulin heavy chain; $\mathrm{V}_{\mathrm{L}}$, variable region of immunoglobulin light chain

\begin{abstract}
Epidermal growth factor receptor (EGFR) is an attractive target for tumor therapy because it is overexpressed in the majority of solid tumors and the increase in receptor expression levels has been linked with a poor clinical prognosis. Also it is well established that blocking the interaction of EGFR and the growth factors could lead to the arrest of tumor growth and possibly result in tumor cell death. A13 is a murine monoclonal antibody (mAb) that specifically binds to various sets of EGFR-expressing tumor cells and inhibits EGF-induced EGFR phosphorylation. We isolated human immunoglobulin genes by guided selection based on the mAb A13. Four different human single chain Fvs (scFvs) were isolated from from hybrid scFv libraries containing a human $V_{H}$ repertoire with the $V_{L}$ of $m A b A 13$ and a human $V_{L}$ repertoire with the $V_{H}$ of $\mathrm{mAb}$ A13. All the $4 \mathrm{scFvs}$ bound to EGFR-expressing A431 cells. One scFv (SC414) with the highest affinity was converted to IgG1 (ER414). The ER414 exhibited $\sim 17$ fold lower affinity compared to the A13 mAb. In addition the ER414 inhibited an EGF-induced tyrosine phosphorylation of EGFR with much lower efficacy compared to the A13 mAb and Cetuximab (Merck $\mathrm{KgaA}$, Germany). We identified that the epitope of A13 $m A b$ is retained in ER414. This approach will provide an efficient way of converting a murine mAb to a human mAb.
\end{abstract}

Keywords: antibody affinity; epitopes; immunotherapy; peptide library; receptor, epidermal growth factor

\section{Introduction}

Epidermal growth factor receptor (EGFR) is a 170 $\mathrm{kDa}$ membrane-spanning glycoprotein comprising an extracellular ligand-binding domain, a transmembrane domain, and an intracellular cytoplasmic protein domain with tyrosine kinase activity (Carpenter, 1987). EGFR belongs to the human epidermal receptor (HER) family of receptor tyrosine kinases, which consists of four closely related receptors - EGFR (HER1, erbB1), HER2 (neu, erbB2), HER3 (erbB3), and HER4 (erbB4) - that mediate cellular signaling pathways involved in growth and proliferation in response to the binding of a variety of growth factor ligands (Yarden and Sliwkowski, 2001; Laskin and Sandler, 2004).

Activation of EGFR has been shown to enhance processes responsible for tumor growth and progression, including proliferation, angiogenesis, invasion and metastasis, and inhibition of apoptosis. Expression of EGFR varies widely in a variety of tumors, including head and neck $(80-100 \%)$, renal $(50-90 \%)$, lung $(40-80 \%)$, breast $(14-90 \%)$, colorectal $(25-77 \%)$, ovarian $(25-70 \%)$, prostate (39$47 \%)$, glioma $(40-63 \%)$, pancreas $(30-50 \%)$ and bladder (31-48\%) (Herbst and Shin, 2002; Capdevila et al., 2009). High levels of EGFR expression in tumors have been correlated with aggressiveness of disease, poor prognosis and decreased survival, poor response to therapy and the development of resistance to cytotoxic agents in some tumor types (Brabender et al., 2001; Capdevila et al., 2009).

The clear potential for EGFR-targeted therapies in the treatment of cancer has prompted the development of a variety of agents targeted to the extracellular ligand-binding domain, the intracellular tyrosine kinase domain, the ligand, or to synthesis of the EGFR (Baselga, 2002; Thomas and Grandis, 2004). Among them, monoclonal antibodies (mAbs) directed against the extracellular domain of EGFR and small-molecule compounds that interfere with intracellular EGFR tyrosine kinase activity are major molecules for therapeutics (Herbst, 2004). 
Previously we reported a generation of novel anti-EGFR mAb A13 (Hong et al., 2010). mAb A13 specifically bound to a variety of tumor cells and efficiently inhibited both EGF-dependant EGFR tyrosine phosphorylation in cervical and breast tumor cells and also in vitro colony formation of EGFR-overexpressing lung cancer cells. Competition and sandwich ELISAs, competitive surface plasmon resonance, and domain-level epitope mapping analyses demonstrated that mAb A13 competitively bound to the domain III (amino acids 302-503) of EGFR with EGF, but recognized a distinct epitope from that of Cetuximab (Hong et al., 2010).

The use of murine antibodies for immunotherapy of human carcinomas has a disadvantage that during the repeated administration of the mAbs to patients, human anti-mouse antibody (HAMA) immune response can be induced. These problems have prompted the use of protein engineering technologies to humanize murine $\mathrm{mAb}$ by transplanting the antigen-binding site from murine to human antibodies, resulting in chimeric or reshaped antibodies. But complete human mAb will be the best choice for the usage in human (Bao et al., 2005).

The idea of guided selection is to use a murine antibody as an epitope template and phage display to drive the selection of human antibodies with corresponding specificity. It is a serial transition from a single murine antibody, via murine-human chimeric forms, to a panel of human antibodies with similar characteristics to those of the starting murine antibody (Osbourn et al., 2005). Several murine mAbs were converted to human antibodies successfully with retention of specificity of original murine mAbs (Beiboer et al., 2000; Klimka et al., 2000; Wang et al., 2000; Schmidt et al., 2001; Kim and Hong, 2007).

Here we report a generation of human mAb to EGFR by guided selection based on the well characterized murine mAb A13. The human scFvs isolated by guided panning were characterized in terms of affinity and binding to EGFR-expressing cancer cells. A fully human IgG1 was constructed from one ScFv which exhibited the highest affinity in all the isolated scFvs, then expressed from Chinese Hamster Ovary ( $\mathrm{CHO}$ ) cells and compared with the mAb A13 and the Cetuximab in regard to affinity and inhibition of EGF-induced tyrosine phosphorylation of EFGR. In addition epitope conservation between the mAb 13 and human $\mathrm{mAb}$ isolated by the guidance of $\mathrm{mAb} A 13$ was proved by competition ELISA.

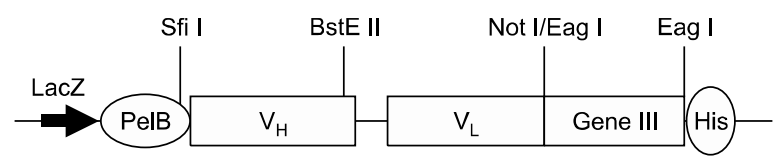

Figure 1. The arrangement of genes in the pSC73His vector. LacZ denotes the lac promoter. PelB denotes leader peptide of pectate lyase $B$ of Erwinia carotovora, Gene III denotes the gene3 that encodes minor coat protein of filamentous phage M13, and His denotes a tag of 6 repeats of Histidine. Restriction enzyme sites used for cloning of scFv are shown.

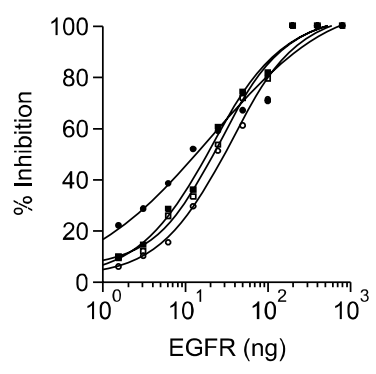

Figure 2. Competition ELISA for measuring relative affinities of anti-EGFR human scFvs which were isolated by guided panning of mAb A13. The inhibition of binding of anti-EGFR human scFvs to EGFR was analyzed on EGFR-coated plates with different concentrations of free EGFR and the curves were fitted with a 4-parameter model using SoftMaxPro software. Each symbol denotes clone number of SC414 (•), SC311 (๘), SC43 ( $\square)$ and SC412(०).

\section{Results}

\section{Construction of A13 scFv and its expression}

DNA sequences of mAb A13 $V_{H}$ and $V_{L}$ were identified previously (Hong et al., 2010). Based on these sequences, primers were designed to incorporate the $\mathrm{V}_{\mathrm{H}}$ and $\mathrm{V}_{\mathrm{L}}$ as scFv to $\mathrm{pSC73His}$ vector (Figure 1) by digesting the $\mathrm{V}_{\mathrm{H}}$ with $\mathrm{Sfi}$ I/BstE II and the $V_{L}$ with BstE II/Not I. The vector has $V_{H}$-linker- $V_{L}$ format with three repeats of $G S$ linker $\left[\left(\mathrm{Gly}_{4} \mathrm{~S}\right)_{3}\right]$ and a $6 \mathrm{XHis}$ tag at the $\mathrm{C}$ terminus of $\mathrm{V}_{\mathrm{L}}$. We identified the soluble expression of ScFv A13 from $E$. coli by ELISA (data not shown).

\section{Selection of human scFvs binding to EGFR}

For human antibody selection by guided panning based on the mAb A13, phagemid vector pSC73His-13V $\mathrm{V}_{\mathrm{H}}-13 \mathrm{~V}_{\mathrm{L}}$ was used to construct phage display libraries with a repertoire of human $V_{L}$ or $V_{H}$. The sizes of resulting hybrid scFv libraries, murine $\mathrm{V}_{\mathrm{H}}$-human $\mathrm{V}_{\mathrm{L}} \mathrm{S}$ and human $\mathrm{V}_{\mathrm{H}} \mathrm{S}$-murine $\mathrm{V}_{\mathrm{L}}$, were $1.5 \times 10^{8}$ and $5.0 \times 10^{8}$ respectively. Then the libraries were panned 5 rounds in EGFR-immobilized immunotubes and the human $V_{H} S$ and human $V_{L} S$ from the $5^{\text {th }}$ round of panning were combined and transformed to E. coli $X L-1$ blue. We found several colonies showing strong binding to 
A

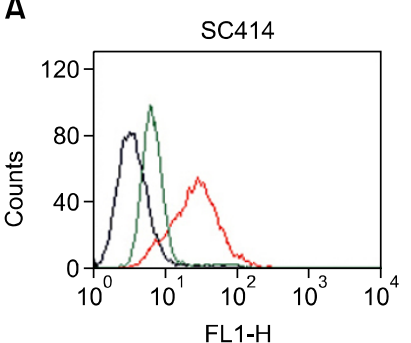

D

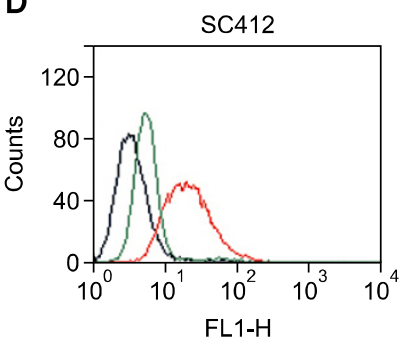

B

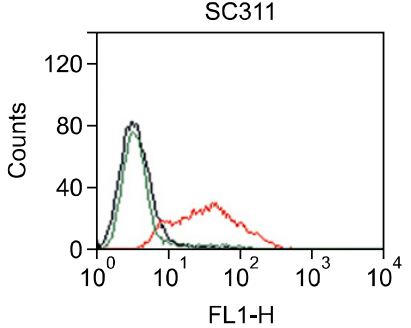

E

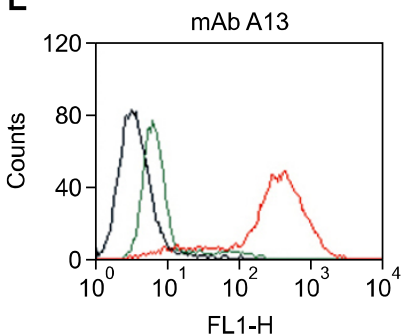

C

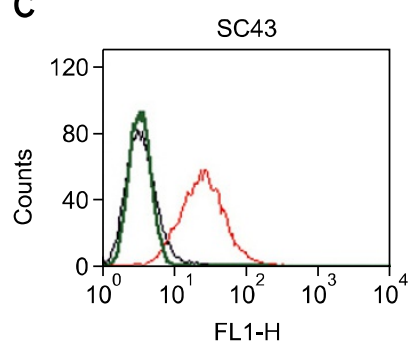

Figure 3. FACS analysis for binding activity of anti-EGFR human scFvs to cell surface-expressed EGFR, in which the scFvs were isolated by guided panning of mAb A13. Differential binding of scFvs to EGFRpositive A431 tumor cells and EGFR-negative HL60 cells (indicated in the panels) was analyzed. Histograms in different color codes represent the reactions of labeled secondary antibody with A431 cells only (black line), scFvs with A431 cells (red line), mAb A13 with A431 cells (red line), and scFvs and $m A b$ A13 with HL60 cells (green lines). A, $B, C, D$ and $E$ denote the FACS diagram of SC414, SC 311, SC43, $\mathrm{SC} 412$ and $\mathrm{mAb} \mathrm{A} 13$ respectively.
EGFR-immobilized ELISA plate and their sequences were identified. Finally we selected 4 different clones. Amino acid sequences of human $\mathrm{V}_{\mathrm{H}} \mathrm{S}$ and $\mathrm{V}_{\mathrm{L}} \mathrm{S}$ from this approach are much similar to that of mouse $\mathrm{mAb} A 13(\gg 90 \%)$. And the $\mathrm{V}_{\mathrm{L}} \mathrm{S}$ are all $\kappa$ chains (sequence data not shown).

In the competition ELISA for comparison of affinities of isolated scFvs, scFv clone 414 (SC414) exhibited highest affinity among 4 different clones although the differences are within 2.5 fold (SC414 $>$ SC $311 \geq$ SC $43 \geq$ SC 412) (Figure 2).

In the FACS analysis to determine whether the scFvs derived from the mAb A13 could bind to cell surface-expressed EGFR, A431 tumor cells which are known to express EGFR with high levels and HL60 cells with no endogenous EGFR expression were employed. We found that all 4 scFvs (SC414, SC 311, SC 43 and SC 412) significantly bound to the A431 cells but not to HL60 cells (Figure 3).

\section{Conversion of ScFv to IgG1and comparison of affinity and signal blocking}

The $V_{H}$ and $V_{L}$ of SC414 were inserted to $p R C-12$ and $\mathrm{pKC}-12$ vectors (Figure 4). The pRC-12 and pKC-12 vectors contain constant parts of human IgG1 heavy and $\kappa$ light chains. Finally a $\mathrm{CHO}$ cell line was established which produces a human anti-EGFR IgG1 $\mathrm{mAb}$ and this was named as ER414. The ER414 mAb was prepared by protein $A$ and ion-exchange chromatography from serumfree culture media and purification was analyzed by SDS-PAGE (data not shown).

In the competition ELISA for comparison of affinities of A13 mAb, ER414 and Cetuximab,
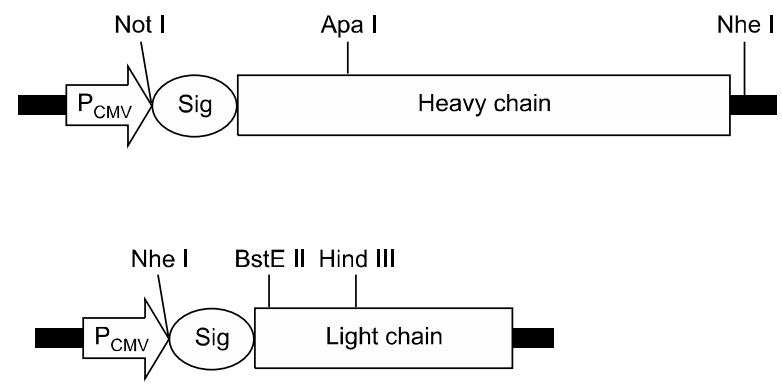

Figure 4. The arrangement of genes in expression vectors of heavy (pRC-12) and light (pKC-12) chains. $P_{\text {CMv }}$ denotes the cytomegalovirus promoter. Sig denotes signal peptide of human immunoglobulin. Restriction enzyme sites used for cloning are shown. The light chain expression vector contains dihydrofolate reductase (dhfr) gene (not shown in this figure) which is used for amplification of integrated genes by adjusting the cells in the increasing concentrations of methotrexate (MTX).

ER414 showed $\sim 20$ fold lower affinity compared to Cetuximab (Figure 5).

Ability of antibody to block the EGF-induced tyrosine phosphorylation of EGFR in MDA-MB-231 cells was compared between ER414 and Cetuximab. Tyrosine phosphorylation of EGFR was induced in the presence of $16 \mathrm{nM}$ of EGF (Figure $6)$. Also there was a basal level of tyrosine phosphorylation of EGFR without EGF (Figure 6). Cetuximab inhibited the EGF-induced tyrosine phosphorylation of EGFR at $\geq 0.67 \mathrm{nM}$ of antibody, whereas ER414 inhibited the EGF-induced tyrosine phosphorylation of EGFR with much higher concentration of antibody (335.0 nM) (Figure 6). In addition A13 mAb showed complete inhibition of EGF-induced tyrosine phosphorylation of EGFR with the concentration of $335 \mathrm{nM}$. 


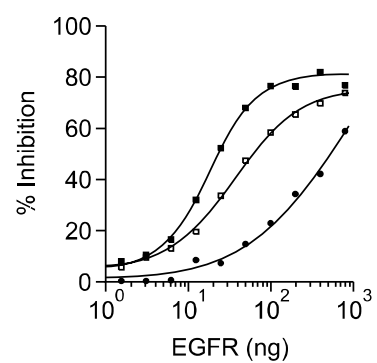

Figure 5. Competition ELISA for comparison of relative affinities of anti-EGFR IgGs. ER414 is a fully human IgG1 originated from the mAb A13 by guided panning. Binding inhibition of anti-EGFR IgGs to EGFR was analyzed on EGFR-coated plates with different concentrations of free EGFR EGFR and the curves were fitted with a 4-parameter model using SoftMaxPro software. Each symbol denotes antibodies of Cetuximab ( $\mathbf{\bullet})$, $\mathrm{mAb} A 13(\square)$ and $\operatorname{ER} 414(\bullet)$.

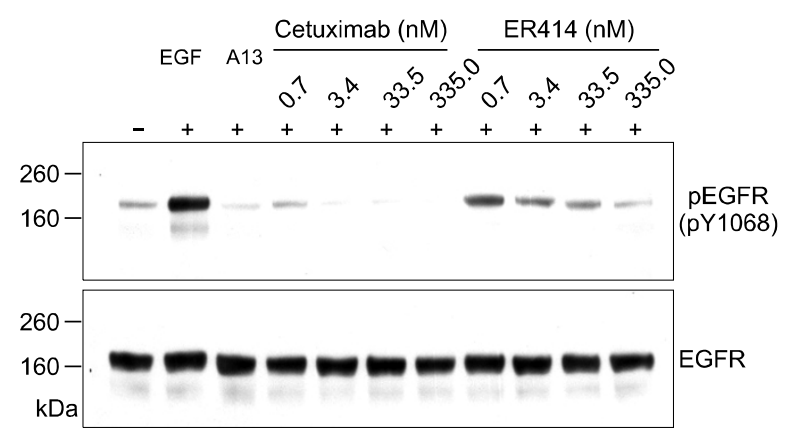

Figure 6. Blocking of EGF-induced tyrosine phosphorylation of EGFR by ER414 and Cetuximab in MDA-MB-231 cells. The serum-starved cells were untreated, treated with EGF ( $16 \mathrm{nM}$ ) only, or treated with EGF (16 $\mathrm{nM}$ ) and different concentrations of either Cetuximab or ER414 for 30 min before the Western blotting analyses. As indicated in the panel 0.7 , 3.4, 33.5, 335.0 nM of Cetuximab or ER414 were included together with $335.0 \mathrm{nM}$ of mAb A13. The expected size of EGFR is $\sim 170 \mathrm{kDa}$.

\section{Epitope conservation of guided-selected mAb}

Epitope conservation of guided-selected $m A b$ was proved by competition ELISA using different epitoperecognizing mAbs $A 13$ and $A 196 . A 13 m A b$ is known to inhibit the EGF-induced tyrosine phosphorylation of EGFR (Hong et al., 2010) and A196 mAb does not inhibit EGF-induced tyrosine phosphorylation of EGFR (unpublished data). Therefore these two mAbs recognize a different epitope of EGFR.

In the competition ELISA, the binding of biotinylated ER414 to EGFR was inhibited by the A13 $\mathrm{mAb}$, indicating that the ER414 recognizes the same epitope with the mAb A13 (Figure 7). The binding of biotinylated ER414 to EGFR was not inhibited by the A196 mAb (Figure 7) which is known to recognize a different epitope. In addition, the binding of biotinylated ER414 to EGFR was inhibited by the ER414 mAb itself (Figure 7). The inhibition efficacy of $A 13$ mAb was better than that

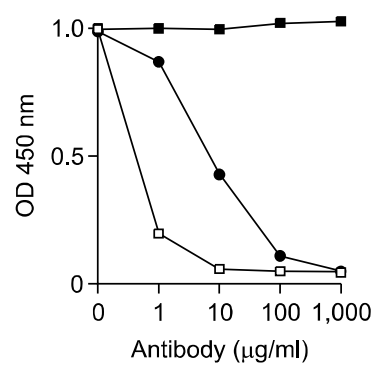

Figure 7. Competition ELISA to analyze whether the ER414 has the same binding site with the murine counterpart $\mathrm{A} 13 \mathrm{mAb}$. The competition was performed on the EGFR-coated plates with $1 \mu \mathrm{g}$ of biotinylated ER414 and different concentrations of free ER414, A13 and A196 mAbs in which free mAbs were employed from $1 \mu \mathrm{g} / \mathrm{ml}$ to $1,000 \mu \mathrm{g} / \mathrm{ml}$. Each

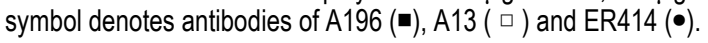

of ER414 due to the higher affinity of $A 13 \mathrm{mAb}$ ( $\sim 17$ fold) compared to ER414.

\section{Discussion}

A method of humanizing a murine mAb is genetic transplantation of the murine complementarity-determining regions (CDRs) into a human framework with closest homology to the present antibody (Jones et al., 1986). To maintain affinity, it is usually necessary to retain additional murine framework-region residues, which can often be laborious and time consuming to identify. The resulting humanized antibody still contains a large amount of murine sequences and there is therefore a risk of immunogenicity (Schmidt et al., 2001).

An alternative method, termed guided selection, has been developed to convert murine antibodies into completely human antibodies with similar binding characteristics. There are two variations of guided selection, "in series" and "in parallel" approaches. In "in series" approach, the murine heavy chain is paired with a human light chain repertoire and selected on antigen. This yields a panel of chimeric antibodies comprising murine heavy-human light. The selected human light chains are then paired with a repertoire of human heavy chains and selected on antigen. Thus, human antibodies that bind to an epitope overlapping with that of the original murine antibody are isolated. However, very few human mAbs by this approach have been reported (Wang et al., 2000; Osbourn et al., 2005).

In "in parallel" approach, the murine light chain is paired with a human heavy chain repertoire while, in parallel, the murine heavy chain is paired with a human light chain repertoire. Both repertoires are then selected on antigen. The resulting selected human heavy and human light chains can then be 
paired together, either individually or as populations, and tested for antigen specificity and function. This method requires a greater technical effort but is likely to provide a wider choice of human antibody candidates compared with the standard "in series" approach (Wang et al., 2000; Osbourn et al., 2005).

In the present investigation, we utilized the "in parallel" approach. Through this approach we isolated 4 different human scFvs and all of them bound to EGFR-expressing A431 cancer cells. In addition all 4 human light chains are $\kappa$ chains although the human light repertoire contained both $\kappa$ and $\lambda$ chains. Same result was found in guided selection of mouse mAb MOPC-31 in which no antibody clones with $\lambda$ chains to a target antigen were obtained. Both the mouse mAb A13 and MOPC-31 which were used as a template for guided selection have $\kappa$ light chains. It is assumed that there was no $V_{H}$ or antigen binding compatible $\lambda$ light chain available in the human $\lambda$ repertoire (Beiboer et al., 2000).

The ER414, a fully human IgG1 derived from the SC414 which showed highest affinity in 4 human scFvs isolated by guided panning, exhibited $\sim 17$ fold lower affinity compared to the originator $\mathrm{mAb}$ A13. There are several reports mentioning similar lower affinity of human clones from guided selection compared to the murine counterpart (Klimka et al., 2000; Wang et al., 2000; Osbourn et al., 2005). Human anti-CD30 mAb which was isolated by guided selection showed 10-fold lower affinity compared to the mouse mAb (Klimka et al., 2000). The lower affinity and as a result lower efficacy of human clones from guided selection require the improvement of affinity. One example is the D2E7, a TNF-a neutralizing human $\mathrm{mAb}$, which was established by stepwise CDR mutagenesis of a human clone from guided selection of MAK 195 (Osbourn et al., 2005).

One of the advantages of guided selection over the direct selection from antibody libraries by phage display is that it makes possible the generation of human mAbs that bind to the same epitope as a conventional murine mAb produced by hybridoma technology (Guo-Qiang and Xian-Li, 2009).

We identified that the epitope of $A 13 \mathrm{mAb}$ is retained in ER414 during guided selection by competitive inhibition of biotinylated ER414 with A13 $\mathrm{mAb}$ in binding to EGFR immobilized ELISA plate. Others reported the retention of epitope specificity between human ScFv from guided selection and murine $\mathrm{mAb}$ which is already characterized the epitope and efficacy (Jespers et al., 1994; Klimka et al., 2000; Figini et al., 2009). And it is the advantage of guided selection that the epitope of well characterized murine $m A b$ is retained in the human counterpart $m A b$.

The ER414 from this investigation is not a product for clinical development. It needs affinity maturation. An article for affinity maturation of ER414 and efficacy studies against EGFR-expressing tumor cells with an affinity-matured mAb is in preparation.

In summary, we successfully isolated a functional human anti-EGFR scFv (SC414) from the murine anti-EGFR mAb A13 by guided selection using phage display technology. A fully human IgG1 (ER414) derived from SC414 retains the epitope specificity of its murine counterpart and inhibits the EGF-induced tyrosine phosphorylation of EGFR but with lower efficiency than the murine counterpart.

\section{Methods}

\section{Construction of scFv A13 and its expression from $E$. coli}

Construction of scFv from hybridoma cells secreting mAb A13 was performed as described previously (Kim et al., 2001a). Briefly $1 \times 10^{8}$ cells were harvested by centrifugation at $1,000 \times g$ and washed 3 times with PBS. Total RNA was prepared using TRIZOL (Life Technologies, Carlsbad, CA) and the cDNAs were synthesized with ImProm- II $^{\mathrm{TM}}$ Reverse Transcription System (Promega, Madison, $\mathrm{WI}$ ) following the manufacture's instruction. Then $V_{H}$ and $V_{L}$ of $m A b A 13$ were amplified by $P C R$ with primers $A 13 V_{H}(F) / A 13 V_{H}(R)$ and $A 13 V_{L}(F) / A 13 V_{L}(R)$ (Supplemental Data Table S1) respectively. Again the PCR DNAs of $A 13 V_{H}$ and $A 13 V_{L}$ were amplified with primers $H$-extension(F)/H-extension( $R$ ) and $\mathrm{K}$-extension( $\mathrm{F} / \mathrm{K}$-extension(R) (Supplemental Data Table S1) and inserted to pSC73His vector (Figure 1) by digestion with Sfi I/BstE II (New England Bio Labs, Beverly, MA) for $V_{H}$ and BstE II/Not I (New England Bio Lab) for $V_{L}$, which was designated as pSC73His- $13 \mathrm{~V}_{\mathrm{H}}-13 \mathrm{~V}_{\mathrm{L}}$. The pSC73His- $13 \mathrm{~V}_{\mathrm{H}}-13 \mathrm{~V}_{\mathrm{L}}$ plasmid was transformed to electrocompetant $E$. coli $X L 1-B / u e$ (Stratagene, La Jolla, CA) by electroporation, and plated on the SB (Super Broth; Kim et al., 2000) agar plates containing $50 \mu \mathrm{g} / \mathrm{ml}$ of carbenicillin. Expression of ScFv A13 from $E$. coli and its measurement by ELISA and sequencing are described below.

Expression of scFv A13 from E. coli was performed in colonies obtained from the SB agar plates as described (Kim et al., 2001a, 2001b; Kim and Park, 2002). Each colony on the SB agar plate was inoculated to $5 \mathrm{ml}$ of SB containing $50 \mu \mathrm{g} / \mathrm{ml}$ of carbenicillin, grown to $O . D_{600}=1$ at $37^{\circ} \mathrm{C}$ with continuous shaking. IPTG (isopropyl- $\beta$-D-thiogalactopyranoside; Sigma, St. Louis, MO) was added to 1 $\mathrm{mM}$, then the bacteria were grown overnight at $30^{\circ} \mathrm{C}$ with continuous shaking. The culture was centrifuged and the supernatant was analyzed for expression by ELISA.

ELISA was performed to measure the binding of expressed scFv to EGFR as described (Kim et al., 2001a, 
2001b; Kim and Park, 2002). In this experiment each well of ELISA plates (NUNC Immuno Module, Maxisorp; Roskilde, Denmark) was coated with $100 \mu \mathrm{l}$ of $2 \mu \mathrm{g}$ EGFR/ml PBS by overnight incubation at $4^{\circ} \mathrm{C}$ and blocked with $300 \mu \mathrm{l}$ of $1 \%$ BSA-PBS by $1 \mathrm{~h}$ incubation at room temperature. Then $100 \mu \mathrm{l}$ of bacterial culture supernatant were added to the plate and incubated for $2 \mathrm{~h}$ at room temperature. Following a washing with PBS-T (PBS buffer containing $0.05 \%$ Tween 20), $100 \mu \mathrm{l}$ of diluted HRP/ Anti-His Tag Conjugate (Qiagen, Valencia, CA) in 1\% BSA-PBS were added and incubated for $1 \mathrm{~h}$ at room temperature. After washing again with PBS-T, $100 \mu \mathrm{l}$ of TMB 2-component microwell peroxidase substrate (KPL, Gaithersburg, MD) were added and the O.D. was measured at $405 \mathrm{~nm}$.

Sequencing of immunoglobulin genes was performed in the Genotech (Daejeon, Korea) with primer Seq001 (Supplemental Data Table S1) and sequence analyses were performed with CLC main workbench (CLC bio, Cambridge, MA).

\section{Construction of hybrid scFv phage libraries}

Hybrid scFv phage libraries containing the mAb A13 $V_{H}$ with human $V_{L}$ repertoire or the $m A b A 13 V_{L}$ with human $V_{H}$ repertoire were constructed as described (Wang et al., 2000; Osbourn et al., 2005). Human $V_{H}$ and $V_{L}$ genes were prepared from total RNAs obtained from human spleen and B cells (Clonetech, Mountain View, CA). cDNAs were prepared using ImProm-II ${ }^{\mathrm{TM}}$ Reverse Transcription System (Promega, Madison, $\mathrm{WI}$ ). The $\mathrm{V}_{\mathrm{H}}$ DNAs were amplified with primers $\mathrm{H}-1 \sim \mathrm{H}-7 / \mathrm{H}-8 \sim \mathrm{H}-10$ (Supplemental Data Table S1), then reamplified with primers $\mathrm{H}$-extension(F)/ $\mathrm{H}$-extension(R) (Table S1). The $V_{L} \kappa$ DNAs were amplified with primers $\mathrm{K}-1 \sim \mathrm{K}-6 / \mathrm{K}-7 \sim \mathrm{K}-9$ (Table 1), then reamplified with primers K-extension( $\mathrm{F}$ )/K-extension(R) (Table 1). The $V_{L} \lambda$ DNAs were amplified with primers $L-1 \sim$ L-9/L-10 L-11 (Supplemental Data Table S1), then reamplified with primers L-extension(F)/L-extension(R) (Supplemental Data Table S1). The $V_{H}$ DNAs were inserted to the pSC73His-13 $\mathrm{V}_{\mathrm{H}}-13 \mathrm{~V}_{\mathrm{L}}$ after digestion with Sfi I/BstE II (New England BioLabs), then the plasmid was introduced to $E$. coli XL1-Blue by electroporation and M13 helper phages were added, and the resulting phage library was designated as pSC73His-hV $V_{H} S-13 V_{L}$. Similarly the $V_{L}$ DNAs which include $V_{L} \kappa$ and $V_{L} \lambda$ were inserted to the pSC73His-13 $\mathrm{V}_{\mathrm{H}}-13 \mathrm{~V}_{\mathrm{L}}$ after digestion with BstE II/Not I (New England BioLabs) and the library was designated as pSC73His-13 $\mathrm{V}_{\mathrm{H}}-\mathrm{h} \mathrm{V}_{\mathrm{LS}}$.

\section{Selection of human scFvs by guided panning}

Panning for selection of phages binding to EGFR was performed in the immunotube (NUNC) as described (Kim and Park, 2002). Tubes were coated with $1 \mathrm{ml}$ of EGFR (Sigma) in $5 \mu \mathrm{g} / \mathrm{ml}$ PBS and blocked with $1 \%$ BSA-PBS. The phages were incubated for $2 \mathrm{~h}$ at $37^{\circ} \mathrm{C}$ in the EGFRcoated tube, then the tube was washed with PBS-T. The phages were eluted from the tube by $0.1 \mathrm{M}$ Glycine buffer ( $\mathrm{pH} 2.0$ ) containing $1 \%$ BSA and neutralized with $2 \mathrm{M}$ Tris. The neutralized phages were infected to $E$. coli XL1-Blue, then the bacteria were infected with M13 helper phage and grown overnight at $37^{\circ} \mathrm{C}$. The phages were prepared using PEG precipitation and used for the next round of panning.

After $5^{\text {th }}$ round of panning, plasmids were prepared from the bacterial pellet and the human $V_{H} S$ and $V_{L} S$ were combined by inserting the human $V_{L} S$ which were cut out with BstE II/Not I (New England BioLabs) from the plasmid of $5^{\text {th }}$ panned pSC73His-13V $V_{H}-h V_{L}$ s to the plasmid from the $5^{\text {th }}$ panned pSC73His-hVHs-13V , which was designated

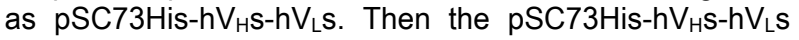
plasmid was transformed to $E$. coli $X L 1-B l u e$ and plated on the SB agar plates containing $50 \mu \mathrm{g} / \mathrm{ml}$ of carbenicillin. Expression of scFv from $E$. coli, ELISA for measuring the binding of SCFv to EGFR, and DNA sequencing were performed as described above.

\section{FACS analysis for ScFv binding to EGFR-expressing cancer cells}

Binding of isolated scFvs to the cell-surface expressed EGFR was analyzed in A431 cells by FACS (FACScan, Becton-Dickinson, Mountain View, CA). HL60 cells, which are known not to express EGFR (Stegmaier et al., 2005), were employed as a negative control. Briefly, $1 \times 10^{7}$ cells were harvested and washed with $1 \%$ BSA-PBS, and incubated with $10 \mu \mathrm{g} / \mathrm{ml}$ of $\mathrm{mAb} A 13$ or scFv from guided selection for $1 \mathrm{~h}$ on ice. After washing with 1\% BSA-PBS, the cells were incubated with FITC conjugated anti-His antibody (Invitrogen, Carlsbad, CA) for scFv or FITC-conjugated goat anti-mouse IgG (Fab specific) (Sigma) for mAb A13 in 1\% BSA-PBS for 40 min on ice, then FACS analysis was performed.

\section{Establishment of a mammalian cell line producing human anti-EGFR IgG}

Each of human $V_{H}$ and $V_{L}$ selected from guided panning was inserted to the mammalian expression vectors $\mathrm{pRC}-12$ (Figure 4) for heavy chain and pKC-12 (Figure 4) for light chain respectively as previously described (Shin et al., 2007). Then the heavy and light chain expression vectors were co-transfected to CHO DG44 cells (originated from Urlaub et al., 1986) using Effectene (Qiagen) and the rest of procedures for screening and selection of cells were followed as described previously (Shin et al., 2007).

Cells producing anti-EGFR antibody were screened by ELISA. Briefly, $100 \mu$ l of EGFR (Sigma) which was diluted to $2 \mu \mathrm{g} / \mathrm{ml}$ PBS were immobilized to each well of ELISA plates (NUNC) and the rest of procedures was followed as described previously (Hong et al., 2010).

\section{Comparison of antibody affinity}

Antibodies were purified using protein $A$ and ion-exchange resins from the culture media. Affinities of antibodies were compared by competition ELISA on the EGFR-coated plates with a free EGFR as described (Kim and Park, 2002; Shin et al., 2007). The EGFR concentration that gives $50 \%$ inhibition of maximum binding (the ELISA reading performed without competitive EGFR) was determined as affinity. 


\section{Inhibition of EGFR tyrosine phosphorylation by mAbs}

To analyze the inhibition of EGFR tyrosine phosphorylation by mAbs, $1 \times 10^{5}$ of MDA-MB-231 cells were plated in 24 well culture plate (NUNC) and serum-starved for $48 \mathrm{~h}$ before the addition of EGF and mAb. After EGF treatment (16 $\mathrm{nM}$ ) with or without $\mathrm{mAb}$ (from $0.7 \mathrm{nM}$ to $335.0 \mathrm{nM}$ ) for 30 min, cells were washed and lysed in TritonX lysis buffer ((Yakes et al., 2002); 10 mM Tris, pH 7.4, 150 mM NaCl, 5 $\mathrm{mM}$ EDTA, $1 \%$ Triton X-100, $2 \mu \mathrm{g} / \mathrm{ml}$ leupeptin \& aprotinin, $1 \mathrm{mM} \mathrm{Na}_{3} \mathrm{VO}_{4}, 1 \mathrm{mM}$ PMSF). The amount of protein was adjusted to be equal $(100 \mu \mathrm{g} / \mathrm{ml})$, boiled with SDS-PAGE sample buffer for $5 \mathrm{~min}$, and SDS-PAGE $(10 \% \mathrm{gel})$ was performed. Then the protein bands were transferred to nitrocellulose membrane (BIO-RAD, Hercules, CA) and probed with rabbit phospho-EGFR (Tyr 1068) antibody (Cell signaling, Danvers, MA) for inhibition of EGFR tyrosine phosphorylation and rabbit EGFR antibody (Cell signaling) for total EGFR and 4CN 2-Component Membrane Peroxidase Substrate Kit (KPL).

\section{Identification of epitope conservation of guided-selected $\mathrm{mAb}$}

Competition ELISA was performed to examine whether the $\mathrm{mAb}$ which was isolated by guided-panning has the same epitope with the counterpart mouse mAb. For this competition ELISA, ER414 mAb which was generated by guided-panning was biotinylated with EZ-Link Sulfo-NHSLC-Biotin (Thermo Scientific, Rockford, IL) by following the instruction from the manufacturer. Then $1 \mu \mathrm{g} / \mathrm{ml}$ of biotinylated ER414 was reacted with different amount of A13 and A196 $(1-1,000 \mu \mathrm{g} / \mathrm{ml})$ on the EGFR-immobilized ELISA plates. A196 is an EGFR binding mAb having a different epitope with A13 (unpublished data). Biotinylated ER414 bound to the immobilized EGFR was detected by Streptavidin-peroxidase polymer, ultrasensitive (Sigma) using TMB microwell peroxidase substrate (KPL).

\section{Supplemental data}

Supplemental data include a table, and can be found with this article online at http://e-emm.or.kr/article/article_files/ SP-44-1-07.pdf.

\section{Acknowledgements}

This work was supported by a grant from Korea Biotech R\&D Group of Next-generation of growth engine project of the Ministry of Education, Science and Technology, Republic of Korea (grant number: 2010K001288) and the Green Cross Corp., Korea

\section{References}

Bao GQ, Li Y, Ma QJ, He XL, Xing JL, Yang XM, Chen ZN. Isolating human antibody against human hepatocellular carcinoma by guided-selection. Cancer Biol Ther 2005;4: 1374-80
Baselga J. Targeting the epidermal growth factor receptor with tyrosine kinase inhibitors: small molecules, big hopes. J Clin Oncol 2002;20:2217-9

Beiboer SH, Reurs A, Roovers RC, Arends JW, Whitelegg NR, Rees AR, Hoogenboom HR. Guided selection of a pan carcinoma specific antibody reveals similar binding characteristics yet structural divergence between the original murine antibody and its human equivalent. J Mol Biol 2000;296:833-49

Brabender J, Danenberg KD, Metzger R, Schneider PM, Park J, Salonga D, Holscher AH, Danenberg PV. Epidermal growth factor receptor and HER2-neu mRNA expression in non-small cell lung cancer Is correlated with survival. Clin Cancer Res 2001;7:1850-5

Capdevila J, Elez E, Macarulla T, Ramos FJ, Ruiz-Echarri M, Tabernero J. Anti-epidermal growth factor receptor monoclonal antibodies in cancer treatment. Cancer Treat Rev 2009;35:354-63

Carpenter G. Receptors for epidermal growth factor and other polypeptide mitogens. Annu Rev Biochem 1987;56: 881-914

Figini M, Martin F, Ferri R, Luison E, Ripamonti E, Zacchetti A, Mortarino M, Di Cioccio V, Maurizi G, Allegretti M, Canevari $\mathrm{S}$. Conversion of murine antibodies to human antibodies and their optimization for ovarian cancer therapy targeted to the folate receptor. Cancer Immunol Immunother 2009;58:53146

Guo-Qiang B, Xian-Li H. Guided selection methods through chain shuffling. Methods Mol Biol 2009;562:133-42

Herbst RS, Shin DM. Monoclonal antibodies to target epidermal growth factor receptor-positive tumors: a new paradigm for cancer therapy. Cancer 2002;94:1593-611

Herbst RS. Review of epidermal growth factor receptor biology. Int J Radiat Oncol Biol Phys 2004;59:21-6

Hong KW, Kim CG, Lee SH, Chang KH, Shin YW, Ryoo KH, Kim SH, Kim YS. A novel anti-EGFR monoclonal antibody inhibiting tumor cell growth by recognizing different epitopes from cetuximab. J Biotechnol 2010;145:84-91

Jespers LS, Roberts A, Mahler SM, Winter G, Hoogenboom $H R$. Guiding the selection of human antibodies from phage display repertoires to a single epitope of an antigen. Biotechnology (N Y) 1994;12:899-903

Jones PT, Dear PH, Foote J, Neuberger MS, Winter G. Replacing the complementarity-determining regions in a human antibody with those from a mouse. Nature 1986; 321:522-5

Kim SH, Titlow CC, Margolies MN. An approach for preventing recombination-deletion of the 40-50 anti-digoxin antibody $\mathrm{V}(\mathrm{H})$ gene from the phage display vector $\mathrm{pComb3.}$ Gene 2000;241:19-25

Kim SH, Chun JH, Park SY. Characterization of monoclonal antibodies against carcinoembryonic antigen (CEA) and expression in E. coli. Hybridoma 2001a;20:265-72

Kim SH, Song SH, Kim YJ, Park SY. Expression and characterization of a recombinant Fab fragment derived from an anti-human alpha-fetoprotein monoclonal antibody. Mol 


\section{Cells 2001b;11:158-63}

Kim SH, Park SY. Selection and characterization of human antibodies against hepatitis $B$ virus surface antigen ( $\mathrm{HBsAg}$ ) by phage-display. Hybrid Hybridomics 2002;21:385-92

Kim SJ, Hong HJ. Guided selection of human antibody light chains against TAG-72 using a phage display chain shuffling approach. J Microbiol 2007;45:572-7

Klimka A, Matthey B, Roovers RC, Barth S, Arends JW, Engert A, Hoogenboom HR. Human anti-CD30 recombinant antibodies by guided phage antibody selection using cell panning. Br J Cancer 2000;83:252-60

Laskin JJ, Sandler AB. Epidermal growth factor receptor: a promising target in solid tumours. Cancer Treat Rev 2004; 30:1-17

Osbourn J, Groves M, Vaughan T. From rodent reagents to human therapeutics using antibody guided selection. Methods 2005;36:61-8

Schmidt A, Muller D, Mersmann M, Wuest T, Gerlach E, Garin-Chesa P, Rettig WJ, Pfizenmaier K, Moosmayer D. Generation of human high-affinity antibodies specific for the fibroblast activation protein by guided selection. Eur J

\section{Biochem 2001;268:1730-8}

Shin YW, Ryoo KH, Hong KW, Chang KH, Choi JS, So M, Kim PK, Park JY, Bong KT, Kim SH. Human monoclonal antibody against Hepatitis $B$ virus surface antigen (HBsAg). Antiviral Res 2007;75:113-20

Stegmaier K, Corsello SM, Ross KN, Wong JS, Deangelo DJ, Golub TR. Gefitinib induces myeloid differentiation of acute myeloid leukemia. Blood 2005;106:2841-8

Thomas SM, Grandis JR. Pharmacokinetic and pharmacodynamic properties of EGFR inhibitors under clinical investigation. Cancer Treat Rev 2004;30:255-68

Wang Z, Wang Y, Li Z, Li J, Dong Z. Humanization of a mouse monoclonal antibody neutralizing TNF-alpha by guided selection. J Immunol Methods 2000;241:171-84

Yakes FM, Chinratanalab W, Ritter CA, King W, Seelig S, Arteaga CL. Herceptin-induced inhibition of phosphatidylinositol-3 kinase and Akt Is required for antibodymediated effects on p27, cyclin D1, and antitumor action. Cancer Res 2002;62:4132-41

Yarden Y, Sliwkowski MX. Untangling the ErbB signalling network. Nat Rev Mol Cell Biol 2001;2:127-37 